\title{
Quaderni
}

QUADERNI Communication, technologies, pouvoir

68 | Hiver 2008-2009

Militantisme médical et fabrique des politiques de santé

Comment la question des enfants « surdoués » estelle devenue scientifiquement sérieuse en France (1971-2007)?

\section{Wilfried Lignier}

\section{(2) OpenEdition \\ Journals}

Édition électronique

URL : http://journals.openedition.org/quaderni/293

DOI : 10.4000/quaderni.293

ISSN : 2105-2956

Éditeur

Les éditions de la Maison des sciences de l'Homme

Édition imprimée

Date de publication : 5 janvier 2009

Pagination : 87-90

\section{Référence électronique}

Wilfried Lignier, «Comment la question des enfants « surdoués » est-elle devenue scientifiquement sérieuse en France (1971-2007) ? », Quaderni [En ligne], 68 | Hiver 2008-2009, mis en ligne le 05 janvier 2012, consulté le 19 avril 2019. URL : http://journals.openedition.org/quaderni/293 ; DOI : 10.4000/ quaderni.293 


\section{comment la question des enfants}

"surdoués"

\section{est-elle devenue} scientifiquement sérieuse en

\author{
France \\ (1971-2007) ?
}

Wilfried

Lignier

CMH-ETT (Ens-Ehess-Cnrs) CSPRP (Université Paris 7)
À partir des années 1970 a commencé à s'affirmer en France une catégorie nosographique socialement remarquable mais longtemps scientifiquement illégitime : la notion de «surdoué ». Directement importée du contexte américain, celle-ci renvoie en particulier aux travaux menés dans l'entre-deux guerres par un important psychologue de Stanford, Lewis Terman ${ }^{1}$; concrètement, elle désigne dès l'origine des enfants ayant obtenu des résultats nettement plus élevés que la moyenne à des épreuves de type psychométrique (on retient en général un seuil de QI global de 130) - ce critère de score étant par ailleurs assorti d'un tableau clinique plus ou moins stable, qui recense en quelque sorte une série de « symptômes » de l'intelligence exceptionnelle chez l'enfant (langage précoce, ennui à l'école, dysgraphie, vivacité des réparties, etc.).

Lorsqu'on s'intéresse de près à l'histoire de cette notion, et en particulier à l'évolution de sa légitimité scientifique, on est tout d'abord frappé par une durable marginalité dans le champ de la psychologie, et plus largement dans le champ scientifique. Jusque dans les années 1980, il n'y pas en France de travaux sur la question: les écrits psychologiques concernant les «surdoués » se résument pour l'essentiel à des textes courts (communications, entrées de dictionnaires, brèves notices dans des manuels de psychiatrie de l'enfant) qui font exclusivement référence aux travaux américains. Cette marginalité dans la littérature peut étonner, dans la mesure où les conditions «techniques» d'une approche proprement scientifique de l'intelligence supérieure chez l'enfant sont réunies de longue date en France : l'échelle métrique de l'intelligence a été inventée par le français Binet, et, au moins 
depuis les années 1950, elle est largement utilisée dans la prise en charge médico-psychologique des enfants. Outre l'importante question de l'impulsion politique, ce paradoxe s'explique sans doute notamment du fait d'un rattachement durable de la question de l' « intelligence supérieure » à une tradition illégitime, celle de l'étude du « merveilleux psychique » - c'est-à-dire d'objets comme la télékinésie, les transmissions de pensée, la médiumnité, etc. - tradition contre laquelle la psychologie n'a justement pas cessé de tenter de se construire ${ }^{2}$. Sur ce dernier point, notons que le livre de 1975 intitulé Les Surdoués. Etudes américaines qui diffusera auprès du grand public, non sans renfort de promotion télévisuelle, le terme même de « surdoués » est publié par Rémy Chauvin, entomologiste au mysticisme avéré (il a par exemple publié en 1967, sous un pseudonyme, un livre intitulé Nos pouvoirs inconnus).

Cette longue marginalité témoigne du fait qu'on ne saurait identifier l'histoire française de l'intérêt scientifique pour l'intelligence enfantine d'exception, à la progressive maturation dans le champ scientifique d'une question qui ne pouvait qu'être présente qu'ab ovo, depuis qu'il est possible de mesurer l'intelligence. Pour comprendre la manière dont le thème a commencé à s'imposer dans l'univers médico-psychologique, il convient en fait de ne pas s'en tenir à une stricte histoire des sciences, et de prendre en compte l'histoire sociale, et en particulier l'histoire des mobilisations politiques. Ainsi se révèlent non seulement la priorité chronologique, mais surtout le rôle déterminant d'un militantisme " pro-surdoué ».

Dès 1971, un psychologue libéral niçois, Jean-
Charles Terrassier, crée, sur le modèle d'associations existant dans le monde anglo-saxon, une Association Nationale Pour les Enfants Surdoués (ANPES) ; à partir de cette date commence un travail de sensibilisation de diverses personnalités politiques, et en particulier des divers ministres de l'Éducation, à la cause des enfants « surdoués », c'est-à-dire à la nécessité de leur offrir une prise en charge scolaire spécifique. Longtemps isolée et inefficace, cette activité de lobbying ne cessera de gagner en importance. Dans les années 19701980, 1'ANPES se renforce symboliquement en participant à la création du Word Council for Gifted Children (1975), en tissant des liens étroits avec des élus locaux, en se rendant visible médiatiquement, ainsi qu'en initiant une approche théorique de sa cause politique : Terrassier propose de comprendre la précocité en termes de «dyssynchronie » du développement enfantin dans Les Enfants surdoués ou la précocité embarrassante (1981). Ce renforcement symbolique finit par rencontrer la bonne volonté de l'État, qui contribue à son tour à une certaine légitimation de la notion - non sans lui faire subir une inflexion terminologique : le Ministère accepte la création de classes spécialisées à Nice en 1987 sous réserve qu'on parle désormais d'enfants « intellectuellement précoces » plutôt que de « surdoués » (du même coup, l'ANPES se fait ANPEIP).

Ce n'est toutefois que dans les années 1990-2000 que le travail de légitimation de la notion nouvelle d' « enfant intellectuellement précoce » va s'accélérer. L'ANPEIP cherche à se développer sur le territoire national ; mais surtout, au milieu des années 1990, une antenne locale de l'association s'autonomise pour devenir l'Association Française pour les Enfants Précoces (AFEP), 
caractérisée par des ressources plus importantes (localisation en région parisienne, fort capital social de la présidente-fondatrice, nombreux adhérents, organisation très structurée), et par conséquent en mesure d'amplifier considérablement le mouvement (notamment grâce à un activisme médiatique d'ampleur). En contact avec des conseillers du ministre Jack Lang, l'AFEP obtient en 2000 la création d'une commission sur la question des enfants précoces, qui rend son rapport final en 2002. Cette date marque un tournant symbolique décisif. Elle scelle une légitimité étatique de la "précocité intellectuelle», qui n'a cessée d'être reconduite jusqu'à aujourd'hui (dans les circulaires de rentrées 2002 et 2003, la loi sur l'Avenir de l'Ecole en 2005, une circulaire spécifique en 2007).

De même que l'ANPES, via son président, a pu se situer sur le terrain scientifique, l'AFEP a depuis sa création cherché le soutien de la science, non pas en produisant quant à elle son propre discours scientifique, mais en s'associant plutôt à des chercheurs extérieurs. Elle s'est ainsi dotée très vite d'un conseil scientifique et a multiplié les invitations de personnalités issues de la recherche à ses manifestations publiques : ainsi, à son congrès de 2006, qui s'est tenu à la Sorbonne, ont pu intervenir un chercheur à l'Inserm, un chercheur au CNRS et un professeur au Collège de France. Ces alliances directement réalisées par les associations contribuent à ce que la cause politique des surdoués se double, récemment, d'une question médico-psychologique de la précocité; elles ne suffisent toutefois pas à rendre compte complètement du développement d'une approche scientifique de la précocité intellectuelle. Il faut en effet expliquer que certains chercheurs qui ne sont pas en contact direct avec les associations prennent aujourd'hui la précocité pour objets et produisent des textes à destination d'un public académique - on note en particulier la parution de numéros spéciaux de revues (Psychologie française, 2004 ; Bulletin de psychologie, 2006) et un manuel universitaire (en 2004). L'intervention de l'État, gagnée par les militants au gré d'un travail politique mené depuis près de 30 ans, a sans doute eu en la matière un effet de conversion symbolique très général, qui excède les interactions directes entre militants et chercheurs ; de même que c'est l'État qui a transformé les « surdoués » en « précoces », c'est l'État qui a fait, pour l'essentiel, de la problématique historiquement indigne de l'intelligence supérieure des enfants une question très sérieuse, que des scientifiques parmi les plus légitimes ont aujourd'hui l'opportunité d'investir. 
$\mathrm{N} \cdot \mathrm{O} \cdot \mathrm{T} \cdot \mathrm{E} \cdot \mathrm{S}$

1. L. Margolin, Goodness personified. The Emergence of Gifted Children, Aldine de Gruyter, 1994.

2. R. Plas, Naissance d'une science humaine: la Psychologie. Les psychologues et le "merveilleux psychique », Presses universitaires de Rennes, 2000. 